\title{
Sulla morte incurabile
}

\author{
Francesco Burrai ${ }^{1}$, Stefano Cabula ${ }^{2}$, Valentina Micheluzzi ${ }^{3}$ \\ ${ }^{1}$ SC Formazione, Ricerca e Cambiamento Organizzativo, ATS Sardegna, Sassari - Italy \\ ${ }^{2}$ Assessorato dell'igiene e sanità e dell'assistenza sociale, Regione Autonoma della Sardegna, Cagliari - Italy \\ ${ }^{3}$ Cardiochirurgia, Azienda Ospedaliero-Universitaria di Sassari, Sassari - Italy
}

\begin{abstract}
On incurable death
Death is incurable. It precedes, transcends and overcomes any possibility of cure. Medicine cures, but leaves man's fundamental event, his death, without cure. After a lifetime of treatment, everyone dies, even those who have used all kinds of treatments, so as not to die. Molecules, surgery, nuclear medicine, technology are not and will never be adequate for death. What can be appropriate for death? Not the escape, but the awareness of its existence, in every minute of the short earthly passage. But how? Man has always used artistic expression, not science, as a way of seeing death. Literature, cinema, theater are human inventions that go through death, and in this article we expose some artistic productions that cure our existence from the "Incurable".
\end{abstract}

Keywords: Cinema, Literature, Death, Theater, Humanism

\section{Introduzione}

II mutamento delle condizioni sociali intervenuto in seguito agli stravolgimenti delle abitudini quotidiane ha indotto le scienze empiriche e quelle umanistiche a incrociare il loro ambito semantico e, quindi, a riflettere su un nuovo concetto di benessere. In particolare laddove la politica deve rispondere a esigenze dell'uomo sotto la sua qualifica di cittadino, la medicina deve rispondere alla domanda dell'uomo nella sua condizione di malato.

Curare il corpo malato significa avere un corpo affetto da una qualche patologia e la medicina persegue il suo scopo nella direzione di una guarigione o di una riduzione o di un controllo della patologia, soprattutto allo scopo di sottrarlo alla morte.

La questione della morte è il centro della questione. Affidare la "cura del corpo" perché lo si sottragga alla morte, soprattutto nei pazienti al termine della loro vita, attraverso la potenza della tecnologia medica, è molto evidente nell'era contemporanea.

\section{Received: October 30, 2020}

Accepted: November 1, 2020

Published online: December 7, 2020

Indirizzo per la corrispondenza:

Dottor Francesco Burrai

SC Formazione, Ricerca e Cambiamento Organizzativo

ATS Sardegna, Sassari - Italy

francesco.burrai@atssardegna.it
Ma la questione non dovrebbe essere posta nei termini di lotta tra tecnologia e morte, lotta nella quale la tecnologia appare l'ausilio esistenziale per allontanare da sé l'idea di una fine, ma, al contrario, la questione dovrebbe essere affrontata in termini di presa di coscienza della propria condizione indipendentemente dalla quantità di tempo che una macchina può "garantire" a un malato al termine della sua vita.

Dunque, il punto è la preparazione personale ad affrontare la morte e a riconoscerla come momento della vita. Questa visione è tipica delle arti, soprattutto in ambito letterario, cinematografico e teatrale. In questo articolo, abbiamo scelto alcune opere che mostrano diverse visioni sull'evento morte.

\section{Modi di morire}

Iona Heath, medico inglese, nel suo testo "Modi di morire" (1), offre una visione chiara del rapporto che si instaura tra medico, infermiere e paziente morente e su come si può affrontare la morte senza negarla. In "Modi di morire" nascono domande fondamentali: come mai la nuova idea dell'affrontare la morte riguarda il morire bendati? Perché è valutata positivamente l'idea di poter morire senza accorgersene? Siamo effettivamente così impreparati all'idea di dover morire al punto da ritenere la morte qualcosa dalla quale fuggire? E soprattutto, perché voler guardare la morte da lontano, come se fosse un evento a noi estraneo e che non ci apparterrà fino a quando non ne saremo coinvolti?

La morte non può essere interpretata come un evento che si scinde dalla vita, le due dimensioni si intrecciano 
inevitabilmente nell'esistenza della persona. La maturazione di un essere umano passa necessariamente attraverso l'idea che, prima o poi, la vita (la data non è concessa a nessuno) avrà un termine. Mantenere un proprio equilibrio psico-fisico passa per la "presa di coscienza" dell'evento morte, la quale non è un'esperienza ma è un momento della vita, da sempre e per sempre. La nostra nascita fa esistere la morte.

Allora, perché proprio in ospedale la dimensione "morte" viene rimessa in discussione alla luce di quelle che sono le più importanti risorse tecnologiche al servizio della scienza medica? Una trasformazione radicale che è entrata nella nostra società, nell'inconscio collettivo, è l'idea del non morire più di vecchiaia, vale a dire senza intervento dell'uomo, ma che la morte, in particolare la morte all'interno degli ospedali, avvenga solo dopo il fallimento di trattamenti terapeutici.

$\mathrm{Ma}$, prima della morte, molto spesso sono presenti il dolore, gli stati d'angoscia. II filosofo Hans George Gadamer ha affrontato il discorso sulla morte e sul morire prendendo in esame il grande tema di come poter condurre e di quanto sia difficile per un sanitario accompagnare la persona alla sua morte. Fino a che punto il sanitario può gestire le sofferenze e, al contempo, non minare la dignità di un uomo sul punto di morire? Come intervenire in questo meccanismo di presa di coscienza, senza sottrarre alla personalità del morente nessuna delle sue acquisizioni e convinzioni personali (2)?

La scienza biomedica, attraverso la farmacologia e la tecnologia, ha preso in mano la condizione della persona che sta morendo, riducendone le sofferenze e il dolore.

Il filosofo Gadamer si chiede, a questo punto, quanto questi interventi impediscano al paziente di vivere lucidamente la propria morte.

L'assistenza con protocolli e procedure è presente nella fase del morire, ma, spesso, i sanitari si nascondono dietro questi strumenti, oggettivi, "freddi", cercando di allontanare gli aspetti soggettivi, "caldi", che pervadono ogni istante di quella fase. Se non ci si nasconde, la morte, allora, non è più un evento sporco, inquietante, ma può essere una fase utile al compiersi del processo di consapevolezza di due persone, l'assistente e l'assistito. Gli ultimi istanti di una persona possono essere un'occasione per spingersi oltre la propria condizione, per tentare di comunicare su ciò che è impossibile conoscere.

Una ricerca del legame e del contatto è una manifestazione di come ci sia la necessità di guadagnare ogni istante con l'altro. Si manifesta, così, l'intenzione di una conoscenza dell'altro, del suo stato, per conoscere il nostro, al limite ultimo della vita.

\section{Parla con lei}

Altro aspetto interessante della relazione sanitariopaziente, sottolineata nel film di Pedro Almodóvar "Parla con lei" (3), è quello relativo al ruolo della speranza nella gestione di un caso estremo. Avere a disposizione la forza di volontà per portare avanti caparbiamente una terapia è un aspetto complesso, alle volte più complesso per chi assiste che per chi è assistito. Nei casi di malati che sono prossimi al morire si tratta di un aspetto piuttosto ambiguo, perché chiama in essere aspetti della dinamica di accettazione ancora più complessi. L'accettare che qualcuno debba morire significa, in realtà, proprio mettere da parte ogni speranza e smettere di credere nel "miracolo" della scienza. Riuscire a nutrire il morente di "normalità" risulta una terapia probabilmente più adeguata del mentire in merito ad aspettative di vita in realtà estremamente ridotte. Ci sono, certamente, aspetti della fase terminale della vita che risultano particolarmente complessi da gestire, come, per esempio, il decadimento del corpo del morente, per il quale si prova una sorta di distacco: un corpo che non reagisce più a stimoli, che non è più in grado di manifestare espressioni, emozioni, percezioni. Un errore di approccio potrebbe, appunto, essere il considerare il morente come una persona che prima "era" in un certo modo, mentre adesso "è" in un modo completamente diverso, irriconoscibile. Dire "non è più lui" di qualcuno che si trova sul punto di morire è un modo, da parte del sano, di prendere le distanze con il problema dell'estinzione di un'esistenza, che viene considerata conclusa ancora prima del fatto reale.

\section{La morte amica}

Marie De Hennezel, psicologa e psicanalista dell'unità di cure palliative presso l'ospedale della città universitaria di Parigi, pratica, con i suoi pazienti, una disciplina ancora poco nota, che è l'aptonomia, una tecnica basata sull'utilizzo del tocco tattile affettivo. Nel suo libro "La morte amica" (4), ci offre una riflessione sull'assistenza sia medica che infermieristica a persone ricoverate nel centro di cure palliative. L'obiettivo è quello di spiegare come il momento della morte possa essere vissuto in maniera completamente diversa, dove la ricerca di un contatto da parte della persona con l'intera equipe ospedaliera è una costante ed è il fulcro per questo centro di cure palliative. Una visione solo oggettiva del mondo crea un mondo in cui si ricerca poco il contatto con l'altro. In un ambito oggettivo come quello medico ospedaliero, dove si "maneggiano" e si "aggiustano" corpi, si dimentica, a volte, ciò che può sentire l'altra persona. La qualità dell'assistenza e il livello di attenzione che imprimiamo in un qualsiasi tipo di cura e di intervento sanitario invasivo o meno, doloroso o sopportabile che esso sia, possono essere attenuati dalla ricerca di un contatto empatico con la persona e, allora, anche l'intervento più aggressivo potrebbe essere percepito diversamente e diventare più tollerato. II tocco del sanitario e il contatto con la persona sono fondamentali per la qualità dell'intervento e il benessere del paziente. Tutti gli interventi di routine assistenziale, dalla mobilizzazione del paziente, all'igiene, alle medicazioni, sono vissuti dai malati molto sofferenti come torture, portandoli a manifestare il desiderio di un'interruzione delle "torture/cure". 
Un esempio di intervento umanizzante, in queste situazioni, è rappresentato dalla collaborazione di due persone, che, essendo in possesso di competenze specifiche, possono "I'una prestare la presenza affettuosa e attenta, mentre l'altra effettua le cure con tutta la competenza necessaria" (4).

\section{La morte di Ivàn II'ìc}

La dimensione del morire è narrata in modo chiaro e lucido da Tolstoj in "La morte di Ivàn II'ic". Nella parte finale del testo, il personaggio soffre per sé, ma riconosce che la sua morte sarà un alleviamento delle pene di chi rimarrà in vita: "Risparmiare loro e risparmiare se stesso da quelle sofferenze" (5).

Il tempo che scorre tra chi sta vivendo la morte, Ivàn, e chi assiste è molto differente.

Il morire come viene descritto in molti testi letterari, così come viene vissuto dai pazienti, si presenta quasi sempre con il sentire una stanchezza infinita, uno sfinimento e nessun orizzonte fino al declino finale del corpo.

Tolstoj, in "La morte di Ivàn II'icc", nel descrivere l'approssimarsi della morte del protagonista, descrive la morte di un uomo colto da una malattia. II grande pensatore Russo considera "normale" la comparsa della malattia nella vita, mentre, in una certa visione medica, viene considerata una complicanza dello stato "normale" della vita. Tolstoj descrive in modo dettagliato gli odori della malattia e quelli del morire, il processo di deterioramento del corpo e l'aria pesante della stanza del malato, che è diversa dall'aria della stanza del sano. Descrive minuziosamente e con abbondanza di dettagli come qualsiasi cosa in quella stanza assuma un'altra forma, un altro odore, indugia sulle riflessioni di chi si accinge a morire e indugia su quelle di chi assiste il morente e attende l'inevitabile momento finale, con lo sgomento di chi si sente del tutto estraneo a quanto sta osservando, come uno spettatore che mai prenderà la parte del protagonista nella tragedia del dialogo tra vita e morte.

Perché dare così tanta importanza al momento della morte? In Tolstoj la morte viene presentata come il climax fatalisticamente predestinato, in cui c'è una relazione tra una certa vita e certi comportamenti, sentimenti, umori, relazioni e una certa specifica morte. Questa idea, che matura entro un contesto religioso e dalle forti connotazioni spirituali, non può che portare il lettore a considerare il momento della morte come il premio o la pena o la conseguenza di una certa vita.

\section{Sette piani}

In un breve racconto di Dino Buzzati, "Sette piani" (6), la negazione della morte avviene attraverso un continuo rimando di possibilità di guarigione, grazie alle avanzate tecniche di cui è fornito l'ospedale.

L'immagine di Giuseppe Corte, il personaggio principale del romanzo, è quella di uomo affannato da una salute altalenante, ma che non influenzerà il corso della sua vita. II suo stato di salute, a detta dei medici, richiede accertamenti specifici, un'eccezionale competenza medica e delle cure e l'assistenza di infermieri altamente specializzati, che si possono trovare solo in uno strano ospedale, bizzarro, a sette piani. L'arrivo del paziente in ospedale è il momento dell'accoglienza, momento fondamentale per una valutazione che è non solo di tipo medico-clinico, ma, soprattutto, relazionale, per stabilire un buon contatto umano con la persona, con una valutazione globale dell'individuo, che si basa sull'osservazione anche del livello di consapevolezza del paziente sul proprio stato di salute. Il contenuto della valigia di Giuseppe Corte è uguale a quello di tutti i pazienti dell'ospedale, che ripongono $i$ propri averi nella stanza di degenza. L'atto di riporre le proprie cose personali rappresenta una speranza, un affidarsi completamente alle competenze, percepite come risolutive, e alla medicina. La medicina che migliorerà il proprio stato di salute fino a una perfetta guarigione. L'ansia e la paura sono scaturite da una discesa all'interno della struttura, e questa discesa è proporzionale al progredire della malattia. La serenità, invece, viene ritrovata ad ogni visita medica oppure durante il colloquio con gli infermieri, che rassicurano il personaggio continuamente sul suo stato di salute, portando alla convinzione che la sua malattia è "as-so-lu-ta-men-te leg-ge-ra".

Questa comunicazione instaura un orizzonte o un'illusione prodotta dalla speranza.

\section{The Kingdom}

II regista danese Lars von Trier, in "The Kingdom" (7), racconta di un anatomopatologo alle prese con i giovani studenti del corso di medicina, che si approcciano allo studio di un cadavere. II cadavere deve essere sezionato. Per l'anatomopatologo l'operazione di dissezione del cadavere implica una specifica sensibilità e una profonda e rispettosa riflessione durante l'atto. Questo approccio è contrario al sentire comune, ovvero di una procedura che potrebbe essere considerata fredda, tecnica e disumana. II medico accoglie gli studenti con un'espressione molto seria e aspetta gli studenti seduto di fronte a una barella, dove si intravede la sagoma di un corpo umano con i soli piedi scoperti. L'espressione molto seria del medico denota il particolare rispetto che merita la situazione. La scena è formata da un'aula con un medico, diversi studenti e un ospite inconsueto: un cadavere. Prima di iniziare la lezione, l'anatomopatologo scopre a mezzo busto il cadavere. Una studentessa esce rispettosamente ma di fretta dall'aula. L'anatomopatologo rispetta la scelta della studentessa, senza commentare in alcun modo la decisione di abbandonare l'aula, e non lasciando spazio a commenti da parti degli altri studenti. L'anatomopatologo chiama uno studente perché lo raggiunga vicino alla barella e lo studente fa un cenno con il capo che manifesta platealmente un "Proprio a me doveva capitare?" ... Lo studente rimane lontano dal cadavere con le mani in tasca. Dice di non avere paura 
del cadavere. Le mani in tasca denotano un'evidente chiusura dello studente rispetto alla possibilità di avere un qualsiasi contatto con il cadavere che gli sta davanti. Lo studente ha mentito: ha paura del cadavere.

II medico, con il suo atteggiamento, conferisce dignità al corpo morto, il quale rappresenta una dimensione umana, uno stato che interesserà tutti, attraverso l'evento morte. In un'aula di anatomopatologia è presente un dialogo di un vivente con la morte, rappresentata dal cadavere: il cadavere parla della morte, della sua azione e del suo risultato sul corpo.

Questa dimensione produce paura, paura di essere toccati. La lontananza dello studente, prodotta dalla paura di essere fisicamente vicino, troppo vicino, al cadavere è una manifestazione di paura della morte e del suo prodotto, come se quel prodotto non dovesse riguardare, prima o poi, anche lui.

Un aspetto interessante è l'eguaglianza morte-collettività. L'anatomopatologo dice: quando cambiamo posto sull'autobus per non essere a ridosso del nostro vicino, di cosa abbiamo paura? Perché cambiamo posto? II contatto con il prossimo, in effetti, provoca, in generale, un senso di fastidio. Esiste tra uomini, una distanza fisica critica, oltrepassata la quale il senso di pericolo e di violazione della propria sfera sembra leso. Ogni volta, prosegue l'anatomopatologo, che un medico indaga con le proprie mani sulla malattia del paziente, sta rompendo quella distanza, quel perimetro difensivo. Se un sanitario evitasse quel contatto o se il paziente evitasse di accettare quel contatto, sarebbe una definitiva vittoria della paura. Nella vita, vincere quella distanza fisica e relazionale dall'altro può condurre a una migliore condizione esistenziale per affrontare la morte degli altri e per l'elaborazione della propria ineluttabile morte.

\section{Il teatro buto}

Un esempio di teatro che affronta il tema della morte in maniera del tutto originale è il teatro buto giapponese (8). L'originalità del modo di spiegare il morire e di farlo fruire al pubblico non consiste nel soggetto della rappresentazione teatrale, che, infatti, non parla di morte, ma che rappresenta il comportamento della morte, come soggetto in azione tra i vivi. Il centro del teatro buto è il corpo dell'attore. II corpo è, attraverso esercizi di preparazione psico-fisica molto stressanti, portato a un estremo dimagrimento, fino al punto di avere nella realtà le sembianze di un cadavere. Primo punto del teatro buto e della rappresentazione della morte operata attraverso il corpo dell'attore è la bruttezza del corpo. Un corpo vivo, di un attore teatrale classico, è un corpo quantomeno armonioso, se non addirittura bello. La morte invece vuole essere rappresentata attraverso la bruttezza del corpo. Sempre sul corpo si concentra la danza dell'attore. I movimenti del teatro-danza buto non sono agili e armonici. L'attore si muove con lentezza. I movimenti obbediscono a un'armonia del tutto estranea a un corpo vivo e vitale. Mettere in scena la morte significa mettere in scena corpi che non sono in grado di gestire la vitalità del movimento come un normale corpo vivo. II primo comportamento dell'attore del teatro buto è la postura. Non rimane mai eretto completamente. L'attore mantiene una postura piegata, le gambe rimangono flesse, quasi storpiate, la schiena è curva. Tutto ciò per manifestare I'incapacità della morte di assumere un contegno tale da passare inosservata tra i vivi.

Che ruolo ricopre, allora, il volto nella danza buto? Esattamente lo stesso che ricopre il corpo. II volto dell'attore viene privato della sua identità ed è coperto di trucco bianco. Il colore è un chiaro riferimento alla colorazione cerulea propria dei cadaveri: il volto non ha alcuna identità ed è la totalità della morte, che non conosce, appunto, identità. Si ha l'espressione dell'abbattimento di ogni singola identità, accomunata, in questa esperienza estrema, dal livellamento di ogni specificità: ogni essere umano è uguale davanti alla morte. II modo di morire è l'esperienza che la morte concede all'uomo, ma diventa irrilevante davanti al fatto morte, esperienza non concessa agli uomini. L'attore buto, allora, con il volto truccato di bianco, annulla la propria identità, per chiudere allo spettatore ogni possibilità di concentrarsi su un qualche aspetto legato all'emotività facciale, a parte quella che avrebbe una certa maschera teatrale. L'attore buto, colorando il proprio volto, assume la maschera della morte e la "recita" senza proferire battute: la morte non ha parole, accade e chiude inesorabilmente la vita, che è piena di parole.

Il teatro buto, dunque, non è parlato. La possibilità comunicativa della morte è solo fisica, non parlata. Gli interlocutori privilegiati di questo dialogo della morte che non parla rimangono il medico o l'infermiere, perché sono coloro che devono tradurre il linguaggio del corpo in una "parola", in una "frase". Per esempio, quando il corpo è troppo caldo, sta parlando e "dice" febbre. Dunque, il linguaggio del morire non può essere parlato, ma solo rappresentato, e il corpo del danzatore buto esprime, dunque, tutto quello che la parola non può proferire. Il cadavere, unico soggetto ad avere compiuto "l'esperienza della morte", non parla, ma ha a disposizione il proprio corpo per comunicare.

L'attore buto assume la portata dirompente del fenomeno morte. II far muovere un corpo morto è un'esperienza scioccante, perturbante. Lo spettatore che fruisce il teatro buto resta molto scosso. Contrariamente al cinema, nel teatro non ci sono effetti speciali, montaggi: rimane lo spettatore da solo, davanti a un attore che ha fatto una scelta di vita radicale, quella di mantenere il proprio corpo in condizioni molto vicine all'anoressia, e lo fa per avere modo di rappresentare un'idea che non può essere confusa con altre: I'idea della morte.

\section{La morte senza cura}

Oggetto di studio della medicina è il corpo. Il corpo è il protagonista assoluto di questa disciplina. Corpo nella 
condizione di malattia, oggetto di studio fondamentale. Obiettivo molto spesso implicito: corpo che non deve morire. Fallimento della disciplina: la morte. Evitare con tutti gli strumenti possibili e con tutta la tecnologia che si ha a disposizione che un corpo malato divenga un corpo morto. Un corpo morto è utile, però, ai corpi vivi, prima che anch'essi diventino corpi morti. Studiare un cadavere può dare indicazioni utili per lo studio delle patologie. Un cadavere può dare organi per altri corpi. Dignità del cadavere: la morte regala alla scienza la possibilità di studiare la vita e la scienza regala, poi, questa conoscenza alla società e la società trae beneficio da qualcosa che teme.

La morte torna a essere un fenomeno sociale, non solo perché interesserà tutti in quanto stato dell'essere, ma anche in quanto stato a disposizione dell'essere per la lotta della conservazione della specie. Per questa ragione, l'anatomopatologo spiega ai suoi studenti che il corpo su quella barella ci appartiene. Occorre studiare i modi di morire e il cadavere come occasione di conoscenza e di dialogo con l'aspetto conclusivo del ciclo della vita.

\section{Conclusioni}

La morte è incurabile. Precede, trascende e supera ogni possibilità di cura. La medicina cura, ma lascia senza cura l'evento fondamentale dell'uomo, la sua morte.

Dopo una vita di cure, tutti muoiono, anche quelli che hanno utilizzato ogni tipo di cura, per non morire. Le molecole, la chirurgia, la medicina nucleare, la tecnologia non sono e non saranno mai adeguate alla morte. Cosa può essere adeguato alla morte?

Non la fuga, ma la consapevolezza della sua esistenza, in ogni minuto del breve passaggio terreste. Ma come? L'uomo ha sempre utilizzato l'espressione artistica, non la scienza, come via per vedere prima della morte, durante e oltre. La letteratura, il cinema, il teatro sono invenzioni umane che passano attraverso la morte, che curano la nostra esistenza dall'Incurabile.

Come nel racconto "Sette piani" di Dino Buzzati, sembra che, davanti all'idea della fine, tutto debba apparire, come se il morire o, meglio, la morte siano una malattia infettiva, contagiosa, che può contaminare come un'infezione nosocomiale ospedaliera. Allora, l'atteggiamento che assumono i sanitari all'interno del racconto di Buzzati è quello di nascondere i moribondi, di negare con la menzogna la reale condizione di progressione della malattia. II vano tentativo che si vuole raggiungere è di eludere il pensiero della morte allontanandola da noi, come se il morire fosse un affare che riguarda gli altri. Per dirla con le parole di Lev Tolstoj: “Ma immediatamente, senza sapere neppure lui come, gli venne in soccorso il solito pensiero che la cosa era capitata a Ivàn Il'ìc e non a lui e che a lui quella cosa non doveva né poteva succedere" (5).

\section{Disclosures}

Conflict of interest: The authors declare no conflict of interest. Financial support: This research received no specific grant from any funding agency in the public, commercial, or not-for-profit sectors.

\section{Bibliografia}

1. Heath I. Modi di morire. Bollati Boringhieri, Torino. 2008.

2. Gadamer HG. Dove si nasconde la salute. Raffaele Cortina, Milano. 1994.

3. Almodóvar P. Parla con lei. Film, Spagna. 2002.

4. De Hennezel M. La morte amica. Grandi Opere, Milano. 1996.

5. Tolstoj L. La morte di Ivàn II'ìc e altri racconti. Oscar Mondadori, Milano. 2010.

6. Buzzati D. Da Il meglio dei racconti di Dino Buzzati, Sette piani. Oscar Mondadori, Milano. 1995.

7. von Trier L. The Kingdom. Film, Danimarca. 1994.

8. Ono K, Ono Y. Nutrimento dell'anima. La danza buto. Aforismi e insegnamenti dei maestri. Ephemeria Edizioni, Macerata. 2015. 